\title{
A novel decellularization method to produce brain scaffolds
}

Alessandro E.C. Granato ${ }^{1,3^{*}}$, Edgar Ferreira da Cruz ${ }^{2 *}$, Dorival Mendes Rodrigues-Junior ${ }^{4 \pi}$, Amanda

Cristina Mosini ${ }^{1}{ }^{\Uparrow}$, Henning Ulrich ${ }^{3}$, Arquimedes Cheffer $^{3}$, Marimelia Porcionatto ${ }^{1 \uparrow}$

${ }^{1}$ Department of Biochemistry, Neurobiology Lab, Escola Paulista de Medicina, Universidade Federal São Paulo, São Paulo, Brazil

2 Department of Medicine, Division of Nephrology, Universidade Federal de São Paulo, São Paulo, Brazil,

${ }^{3}$ Department of Biochemistry, Instituto de Química, Universidade de São Paulo, São Paulo, Brazil

${ }^{4}$ Department of Biological Sciences, Universidade Federal de São Paulo, Diadema, Brazil

\section{${ }^{*}$ Corresponding authors}

E-mail: alessandroecgranato@gmail.com and edgarferreiracruz@yahoo.com.br

IThese authors contributed equally to this work

\&These authors also contributed equally to this work 


\section{ABSTRACT}

Scaffolds composed of extracellular matrix (ECM) can assist tissue remodeling and repair following injury. The ECM is a complex biomaterial composed of proteins, glycoproteins, proteoglycans, and glycosaminoglycans, secreted by cells. The ECM contains fundamental biological cues that modulate cell behavior and serves as a structural scaffold for cell adhesion and growth. For clinical applications, where immune rejection is a constraint, ECM can be processed using decellularization methods intended to remove cells and donor antigens from tissue or organs, while preserving native biological cues essential for cell growth and differentiation. Recent studies show bioengineered organs composed by a combination of a diversity of materials and stem cells as a possibility of new therapeutic strategies to treat diseases that affect different tissues and organs, including the central nervous system (CNS). Nevertheless, the methodologies currently described for brain decellularization involve the use of several chemical reagents with many steps that ultimately limit the process of organ or tissue recellularization. Here, we describe for the first time a fast and straightforward method for complete decellularization of mice brain by the combination of rapid freezing and thawing following the use of only one detergent (Sodium dodecyl sulfate (SDS)). Our data show that using the protocol we describe here the brain can be entirely decellularized, while still maintaining ECM components that are essential for cell survival and repopulation of the scaffold. Our results also show the repopulation of the decellularized brain matrix with Neuro2a cells, that were identified by immunohistochemistry in their undifferentiated form. We conclude 
that this novel and simple method for brain decellularization can be used as a biocompatible scaffold for cell repopulation.

Key Words: decellularization; brain; scaffold

\section{Impact Statement}

For the first time we describe an easy, effective and low cost method for complete decellularization of murine brain by the use of only one detergent (SDS) combined with rapid freezing and thawing, that can be used as a 3D scaffold for cell culture of neuronal cells. The results show that the decellularized brains still maintain ECM components essential for cell survival and repopulation of the scaffold. Moreover, we found that the decellularized brain matrix can be repopulated with neural cells, showing its biocompatibility.

\section{INTRODUCTION}

Chronic diseases represent a burden to the individuals as well as to the health systems worldwide. Recently, several strategies have been developed to better understand and eventually treat chronic diseases. These strategies comprehend the establishment of organoids produced from human induced pluripotent stem cells (hiPSC), bioengineered tissues, and decellularized scaffolds from lungs, hearts, livers, and kidneys ${ }^{(1-5)}$. Despite the great advances till now, none of these processes can reproduce with high fidelity the full complexity of a tissue or organ. Combinations of organoids and organ-on-a-chip technology have been proposed, but still face technical difficulties, including the correct assemble of a given tissue microenvironment ${ }^{6}$. Thus, one of the most challenging aspects of tissue engineering is to reproduce the correct disposition 
of the extracellular matrix (ECM), known not only to support correct cell biology processes, but in many cases, to drive them. The brain is an emblematic example of this issue and the production of brain organoids from hiPSC has been described in the literature by several laboratories (7-10). Interestingly, the organoids present gene expression that recapitulates human cortical development, indicating that the maturation of a human organoid runs in synchrony with healthy human fetal development ${ }^{11}$. Despite the power of organoid cultures, there are some limitations such as the lack of full organ organization and orientation. Furthermore, there is a size limitation to organoids as they are not vascularized in vitro $(12,13)$.

The solution may lay in the reproduction of the ECM composition, not only with regard to the types of proteins, glycoproteins, and proteoglycans, but also to their arrangement in the tissue ${ }^{(14-21)}$. Decellularization of organs can produce ECM scaffolds that can be used for stem cell differentiation as an alternative to organoids. The ECM acts as a support for cells during embryo development, induces cell differentiation and proliferation, promotes cell adhesion and cell migration, and provides cues to maintain tissue homeostasis and regeneration. Similar to organoids, acellular organ substrates combined with patient-derived stem cells can create a platform for personalized medicine studies ${ }^{13}$. Properly decellularized organs retain both the structural proteins and biologically active growth factors that are capable of inducing location-specific differentiation of stem and progenitor cells $(13,22,23)$.

Tridimensional (3D)-printed scaffolds are an attractive alternative to decellularized scaffolds. However, it is currently difficult to use this technology to replicate the intricate ECM structure of complex organs, such as the brain, and 
the composition of region-specific growth factors on the ECM scaffold ${ }^{13}$. To produce a bioscaffold, several steps are required in order to achieve complete decellularization and that can be performed by flushing detergents or enzymes through to the main artery of the organ. Based on this, several protocols have been described, but none of them has been shown to be more effective than other. Moreover, the total removal of cellular and nuclear components is crucial, as cellular or subcellular fragments left can trigger an immunological response. On the other hand, biological activity of the remaining ECM and preservation of the 3D architecture of the tissue, including a wide microvascular system, must be maintained (24-30).

Generally, two different approaches for cellular repopulation have been described: (i) infusion of cells via the conserved vascular network and (ii) direct injection of cells into the $\mathrm{ECM}^{(30-32)}$. In the present study, we chose the second approach due to the significant reduction of the brain size after complete decellularization. Hence, we proposed a new method for decellularized brain scaffold production that could be used as a template for tissue bioengineering as the ECM structure is preserved.

\section{MATERIAL AND METHODS}

\section{Experimental Model}

Mice (Mus musculus, C57/BI6 lineage) weighting 25-30 g, were obtained from the University's Animal Facility (CEDEME/UNIFESP). All protocols were approved by the University's Committee of Ethics in the Use of Animals (CEUA \#2101180516). Every effort was made to minimize animal suffering and reduce the number of animals used. For the surgical proceedings, mice were 
anesthetized with Ketamine $75 \mathrm{mg} / \mathrm{Kg}$ and Xylazine $10 \mathrm{mg} / \mathrm{Kg}$ by intraperitoneal injection. Trichotomy and skin antisepsis were performed in abdominal site with chlorhexidine-alcohol and sterile cotton gauzes. Mice used to harvest the brain were placed in a surgical table at $37^{\circ} \mathrm{C}$ in dorsal decubitus.

\section{Decellularization process}

Brains were obtained from adult C57BL/6 mice, placed inside a $2 \mathrm{~mL}$ tube and soaked in liquid nitrogen for $10 \mathrm{~min}$. After this rapid freezing process, the brains were thawed at room temperature and washed with $10 \mathrm{mM}$ PBS for $5 \mathrm{~min}$. This freeze-thawing process was done 3 times. After this, each brain was soaked in a $10 \mathrm{~mL}$ solution of $1 \%$ SDS, and agitated for $24 \mathrm{~h}$. The decellularized brains were washed 3 times with $10 \mathrm{mM}$ PBS for $5 \mathrm{~min}$.

Residual nucleic acid and protein quantitation

Nucleic acid quantitation was performed in samples of $100 \mathrm{mg}$ of decellularized matrix or control fresh tissue, triturated in $1 \mathrm{~mL}$ Trizol (Life Technologies, USA) using a Polytron Pt 1200 Cl homogenizer (Kinematica AG, Switzerland). One $\mathrm{mL}$ of homogenate was transferred to propylene tubes and $250 \mu \mathrm{L}$ of chloroform were added. After, tubes were sealed, homogenized and centrifuged $10000 \times \mathrm{g} ; 15 \mathrm{~min}$ at $4^{\circ} \mathrm{C}$. The supernatant was discarded and $300 \mu \mathrm{L}$ of ethanol were added, and the mixture was gently homogenized in vortex and incubated at room temperature for $3 \mathrm{~min}$. The solution was centrifuged $10000 \times \mathrm{g}$; $5 \mathrm{~min}$ at room temperature. The DNA pellet was washed twice with $0.1 \mathrm{M}$ citrate buffer and suspended in $1 \mathrm{~mL}$ of $75 \%$ ethanol, incubated for $15 \mathrm{~min}$ with homogenization. The DNA solution was centrifuged at $10000 \times \mathrm{g} ; 5 \mathrm{~min}$ at room 
temperature and the supernatant was gently removed, the pellet was dried for 5 min under vacuum and $100 \mu \mathrm{L}$ of DNase free water were added.

DNA and RNA were isolated from native and decellularized tissue to perform the nucleic acid quantitation. The samples were triturated in $1 \mathrm{~mL}$ of Trizol using a Polytron Pt $1200 \mathrm{Cl}$ homogenizer (Kinematica AG, Littau, Switzerland). One $\mathrm{mL}(1 \mathrm{~mL})$ of homogenate was transferred to propylene tubes and $250 \mu \mathrm{L}$ of chloroform were added. After, the tubes were sealed, homogenized and centrifuged $10,000 \mathrm{xg}$, for $15 \mathrm{~min}$ at $4^{\circ} \mathrm{C}$. The supernatant was discarded and $300 \mu \mathrm{L}$ of ethanol were added, gently homogenized and incubated at room temperature for $2-3 \mathrm{~min}$. Centrifuged at $10,000 \mathrm{xg}$, for $5 \mathrm{~min}$ at room temperature. DNA pellet was washed twice with $0,1 \mathrm{M}$ citrate buffer and suspended in $1 \mathrm{~mL}$ of $75 \%$ ethanol, incubated for 15 min with periodic homogenization, centrifuged at $10,000 \times g$, for $5 \mathrm{~min}$ at room temperature and the supernatant was gently removed, dried for 5 min in vacuum and $100 \mu \mathrm{L}$ of DNase free water were added. Nucleic acid content was quantified using NanoDrop ND-1000 (Thermo Fisher Scientific, USA). DNA and RNA contents were normalized to the initial dry weight of the samples. Base pair length of residual DNA was determined.

The total protein of the decellularized and non-decellularized brains was extracted using Mammalian Cell Extraction Kit (Biovision, USA) following the recommendations of the manufacturer, and after that the protein was quantified with Qubit (Thermo Fisher Scientific) fluorimeter.

\section{Characterization of decellularized tissues constituents}

Decellularized and fresh tissues were fixed in $4 \%$ paraformaldehyde and embedded in paraffin. Tissue sections $(5 \mu \mathrm{m})$ were obtained and used for 
histological analysis. Haematoxylin and eosin (H\&E) staining was performed according to standard protocol to evaluate the tissue morphology, specially the absence of residual cellular nuclei in the decellularized tissues, also confirmed by 4',6-diamidino-2-phenylindole fluorescent dye (DAPI, 1:1000, Molecular Probes, USA) staining.

Basal membrane was evaluated by Periodic Acid-Schiff (PAS) staining, and Masson's trichrome staining was used to stain collagen fibers. Alcian blue staining was used to evaluate the presence of sulfated glycosaminoglycans and picrosirius red was used to evaluate the presence of collagens type I, II, and III. All reagents used for the histological analysis were purchased from SigmaAldrich (USA), and the images were acquired with an Olympus BX61 microscope (USA).

Culture of Neuro2a (mouse neuroblastoma)

Neuro2a cells were cultured according to the recommended protocol from ATCC (American Type Culture Collection, USA). Briefly, cells were cultured in a $75 \mathrm{~cm}^{2}$ culture bottle with $15 \mathrm{~mL}$ of culture medium composed of high glucose DMEM (Dulbecco's Modified Eagle's Medium, GibcoBRL, USA), 1 \% penicillin / streptomycin (GibcoBRL), $1 \%$ glutamine (Sigma-Aldrich) and $10 \%$ fetal bovine serum (FBS; Cultilab, Brazil). Cells were kept in an incubator at $37^{\circ} \mathrm{C}$ and $5 \% \mathrm{CO}_{2}$. Upon reaching $60 \%$ confluence cells were trypsinized and subcultured in the ratio of $1: 5$.

Immunostaining 
The control brains or decellularized brains were immunolabeled with antilaminin antibody (Merck Millipore, USA), followed by incubation with fluorophoreconjugated secondary antibody (Alexa 488 or 594; Molecular Probes, USA) plus DAPI. Coverslips were assembled with Fluoromount G (Electron Microscopy Sciences) and then analyzed using an inverted confocal microscope (Leica Microsystems, Germany). Image overlays were generated using ImageJ software (NIH, USA).

Neuro2a cells were injected on decellularized brains and kept in an incubator at $37^{\circ} \mathrm{C}$ and $5 \% \mathrm{CO}_{2}$. After this period, cells were fixed with $4 \%$ paraformaldehyde, permeabilized with $0.1 \%$ Triton X-100 (Sigma-Aldrich), and immunollabeled with anti-beta III tubulin (Tubbeta3) (Merck Millipore) followed by incubation with fluorophore-conjugated secondary antibody (Alexa 488 or 594) plus DAPI. Coverslips were assembled with Fluoromount G (2: 1 in PBS) and then analyzed by an inverted confocal microscope. Image overlays were generated using ImageJ software.

Repopulation of decellularized brain with Neuro2a cells

Repopulation of decellularized brain was performed after sterilization of the tissue with peracetic acid (PAA) 1:1000 in $20 \%$ ethanol, placed on 24-well culture plate. After washing the samples 6 times with high glucose DMEM, $1 \times 10^{6}$ Neuro2a cells were injected into the decellularized brain, and culture medium was added, until the incubation time periods were reached ( 24 and $72 \mathrm{~h}$ ). After these periods of time, the repopulated decellularized brain was analyzed. 
Samples of repopulated and non-repopulated decellularized brain fixed in $2.5 \%$ glutaraldehyde solution buffered in $0.1 \mathrm{M}$ sodium cacodylate solution, $\mathrm{pH}$ 7.2. After the fixation process, samples were washed with $0.1 \mathrm{M}$ sodium cacodylate buffer, $\mathrm{pH} 7.2$ and submitted to metallic impregnation. To make this, samples were incubated in $2 \%$ osmium tetroxide in $0.1 \mathrm{M}$ sodium cacodylate buffer, $\mathrm{pH} 7.2$ for $2 \mathrm{~h}$, washed with $0.1 \mathrm{M}$ sodium cacodylate buffer $\mathrm{pH} 7.2$ three times during $15 \mathrm{~min}$, and incubated in $1 \%$ tannic acid water solution for $45 \mathrm{~min}$, followed by two washes in distilled water (10 $\mathrm{min}$ each). After metallic impregnation, samples were dehydrated gradually in 50-70-90 \% ethanol (twice, 30 min each) and $100 \%$ (three times, 30 min each) and then, samples were submitted to a drying process in a critical point chamber (Balzers CPD 030, Lichtenstein) using $\mathrm{CO}_{2}$. Samples were then coated with a thin layer of $20-30 \mathrm{~nm}$ thickness of gold (Sputtering, Leica Microsystems, Germany) and scanned on a FEI Quanta 250 FEG scanning electron microscope (ThermoFisher, USA).

\section{Statistical Analysis}

The data were analyzed using the Statistical Package for Social Sciences (SPSS version 23.0, USA). The results were expressed by average \pm SEM, and significance was tested by $t$-test or one-way ANOVA, as appropriate. The value of $p<0.05$ was considered significant.

\section{RESULTS}

Murine brains were fully decellularized by SDS 
After fast freezing in liquid nitrogen followed by $24 \mathrm{~h}$ in $1 \%$ SDS, the mice brains were analyzed to investigate the remaining of cells, nucleic acids and proteins. Figure $1 \mathrm{~A}$ presents a representative image showing that decellularized the brain became smaller, with less blood after the decellularization procedure. Further direct confocal microscopy analysis on a decellularized mouse brain section revealed the absence of nuclei stained with DAPI (Figure 1D), as compared to the clear presence of DAPI-stained nuclei in fresh nondecellularized mouse brain (Figure 1B). The absence of nuclei was confirmed by $\mathrm{H} \& \mathrm{E}$ staining (Figure $1 \mathrm{C}$ and $\mathrm{E}$ ). Therefore, the fully decellularized murine brains were then named decellularized brain matrix (DBM).

\section{Decellularization reduces the amount of nucleic acids leaving a higher protein concentration per weight of DBM}

The quantification of DNA, RNA, and protein was performed in order to evaluate the effectiveness of decellularization. DNA, RNA, and protein concentrations were normalized by brain weight. The amount of DNA (Figure 1F) and RNA (Figure 1G) decreased after the decellularization process, whereas the the total protein concentrations was statistically higher after decelularization (Figure $\left.1 \mathrm{H},{ }^{*} \mathrm{p}=0.0155\right)$. 

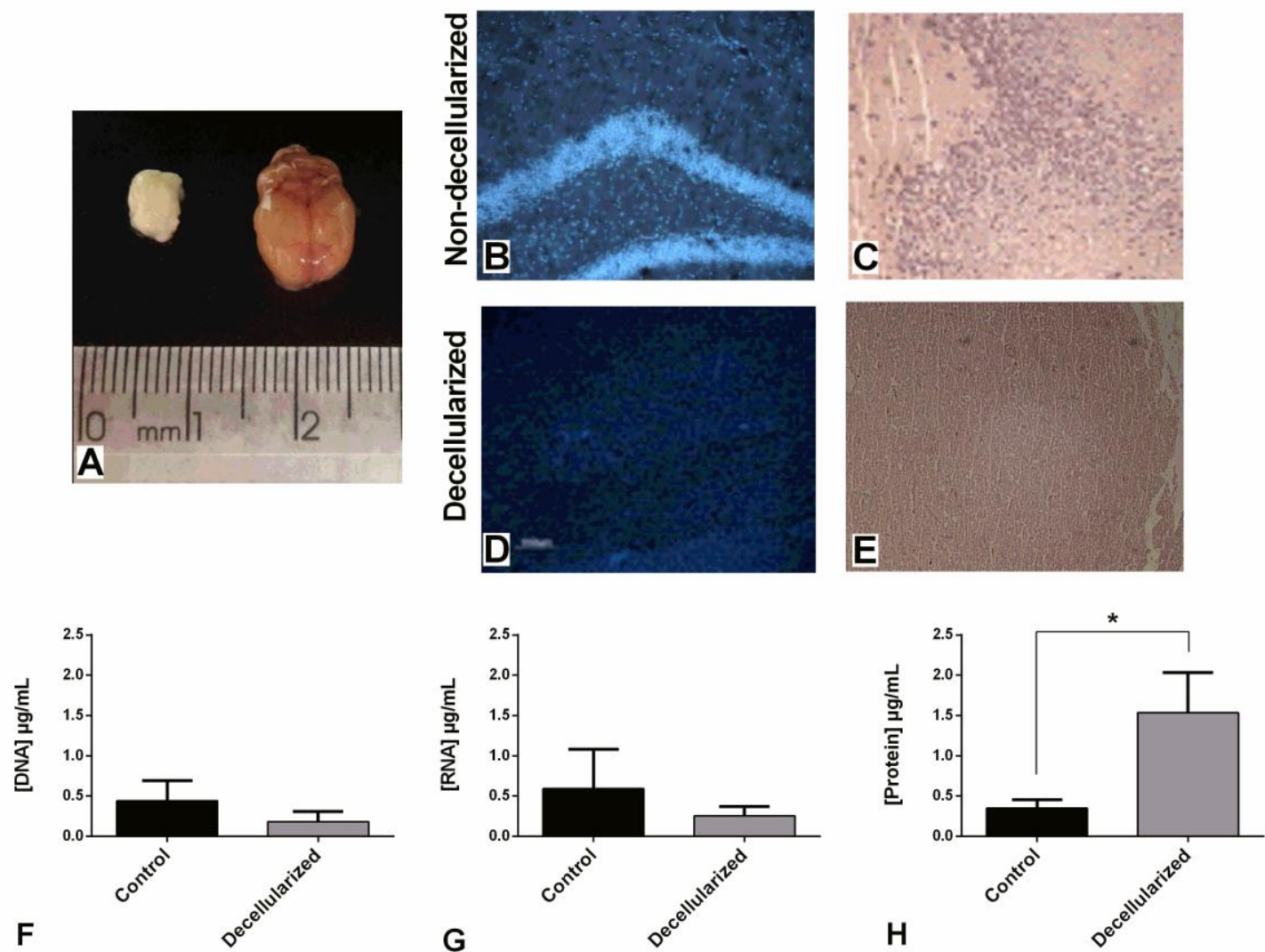

Figure 1: (A) Morphology change of the decellularized brain (left) and non-decellularized brain (right). Representative DAPI staining on $2 \mu \mathrm{m}$ cryosections obtained from a non-decellularized (B) and a decellularized (D) mouse brain section. Representative haematoxylin-eosin (H\&E) staining on $2 \mu \mathrm{m}$ cryosections obtained from a non-decellularized $(C)$ and a decellularized $(E)$ mouse brain section. Scale $=200 \mu \mathrm{m}$. DNA $(F)$ and RNA $(G)$ quantification showed lower concentrations of nucleic acids in the decellularized scaffold compared with the nondecellularized tissue (Control), when both normalized with their dry weight. Protein quantification $(\mathrm{H})$ showed higher concentrations of protein in the decellularized scaffold compared with the nondecellularized tissue (Control), when both normalized with their dry weight. T student test: ${ }^{*} p<$ 0.05 .

\section{DBM maintains essential ECM proteins after decellularization}

Alcian Blue stains acidic polysaccharides such as glycosaminoglycans

(Figures 2A and B). Masson's Trichrom staining of collagen IV shows that its architecture was not affected by decellularization protocol (Figures $2 \mathrm{C}$ and D). Periodic Acid-Schiff (PAS) is a staining method used to detect polysaccharides such as glycogen, and glycoproteins, glycolipids and mucins in tissues. PAS staining showed that although the decellularization process was performed with a detergent (SDS), the basal lamina remained preserved (Figures $2 \mathrm{E}$ and $\mathrm{F}$ ). 
Hematoxylin and eosin (H\&E) staining is used to stain cell nuclei in tissues and to control reinforcing the effectiveness of decellularization process (Figures 2G and $\mathrm{H})$.

$A B$
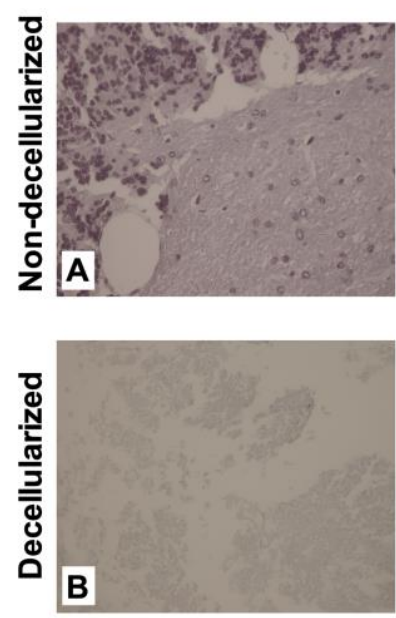

MT
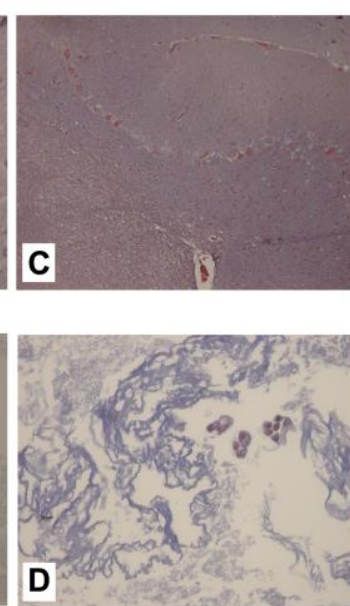

PAS
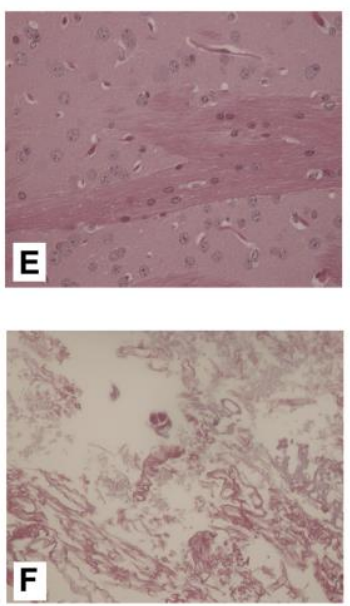

H\&E

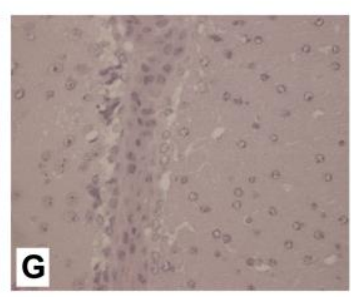

H

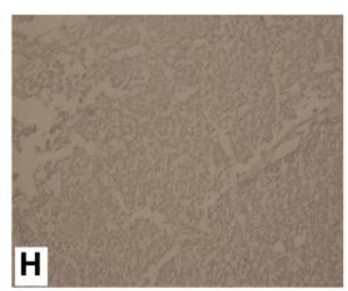

Figure 2: Representative Alcian Blue staining (A) and (B). Representative Masson's Trichome $(C)$ and $(D)$. Representative PAS staining $(E)$ and $(F)$. Representative H\&E staining $(G)$ and $(H)$. Cryosections obtained from a non-decellularized (Above) and a decellularized (Below) mouse brain section. Scale $=50 \mu \mathrm{m}$.

\section{DBM maintain collagen and laminin after decellularization}

The immunofluorescence performed in non-decellularized brains and in DBM identified the presence of laminin, a protein crucial for the repopulation process. The results showed the presence of laminin (Figures $3 \mathrm{~A}$ and $3 \mathrm{~B}$ ). Picrosirius red staining was performed to observe the presence of collagen type I, II and III (Figures 3C and 3D). Under polarized light, collagen bundles appear green, red, or yellow, and are easily differentiated from the black background ${ }^{33}$. These two assays showed that laminin and collagen type I, II, and III were also preserved after the decellularization process. 


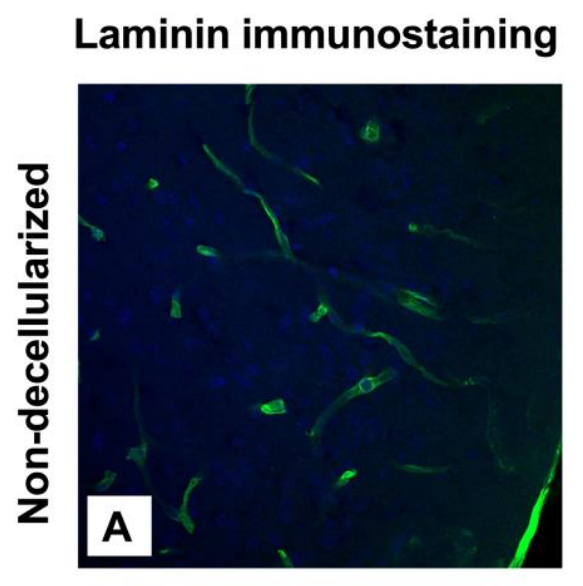

\section{Picrosirius red staining}
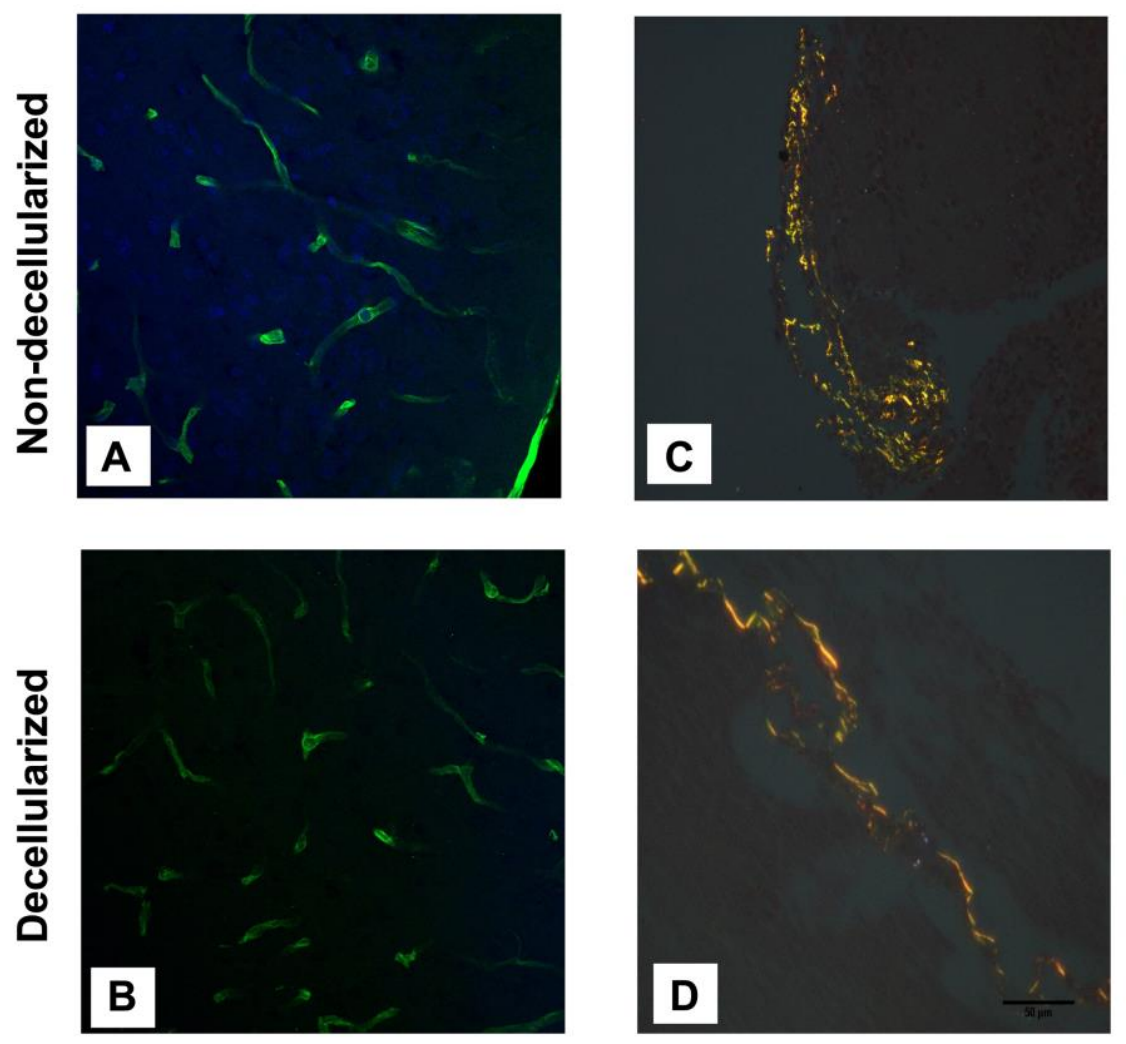

Figure 3: Representative Laminin immunostaining (green) with nuclear staining DAPI (blue) of non-decellularized (A) and decellularized (B) mouse brain section. Representative Picrosirius Red staining on $2 \mu \mathrm{m}$ cryosections obtained from a non-decellularized (C) and a decellularized (D) mouse brain section. Scale $=50 \mu \mathrm{m}$.

\section{SEM microscopy shows the architecture of the DBM repopulated with}

\section{Neuro2a cells}

We also aimed to analyze if Neuro2a cells adhered when grown on DBM as they would do while cultured on plastic or glass. SEM analysis of slices of the internal region of DBM showed the presence of fibers of different lengths and thicknesses (Figure 4A) and holes that appear to be ripped cell nuclei (Figure 4B) from inner sections. In slices of the internal region of the DBM we could also observe the preservation of grooves (Figure 4E) while in the visualization of the external region of the DBM we could notice the presence of a vessel (Figure 4F). After recellularization for $24 \mathrm{~h}$ we observed that cell spreading and extended 
bioRxiv preprint doi: https//doi.org/10 1101/680702; this version posted June 24 2019. The copyright holder for this preprint (which was not certified by peer review) is the author/funder, who has granted bioRxiv a license to display the preprint in perpetuity. It is made available under aCC-BY-ND 4.0 International license.

cellular protrusions were rare (Figure $4 \mathrm{C}$ and D), suggesting that there was no evidence of neuronal differentiation of Neuro2a cells, indicating that during this period of incubation the scaffold did not induce neuronal differentiation.

Decellularized
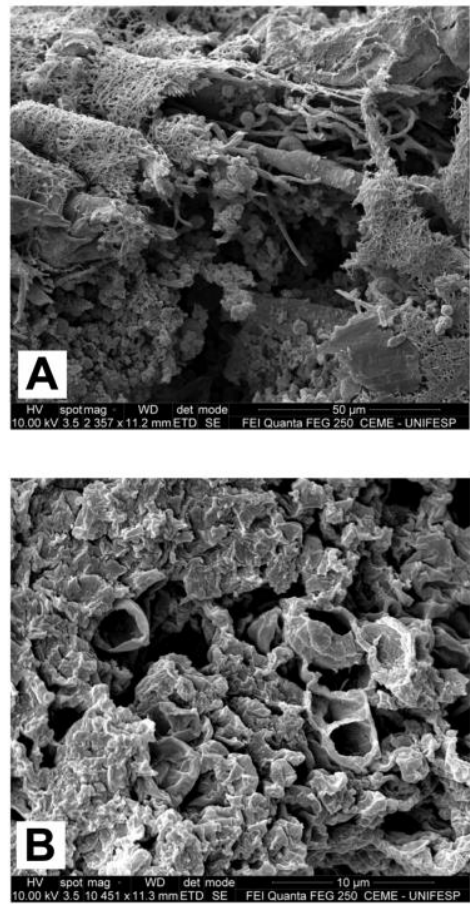

Decellularized + Neuro2a
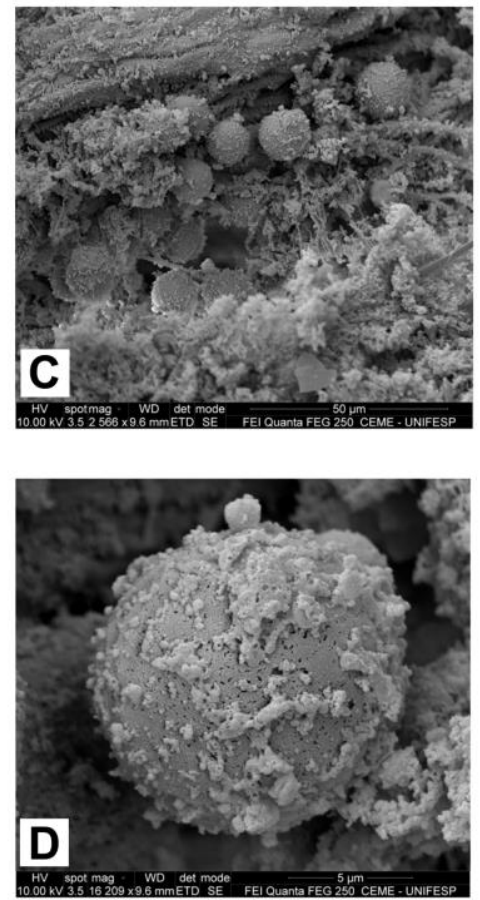

Decellularized Innerface

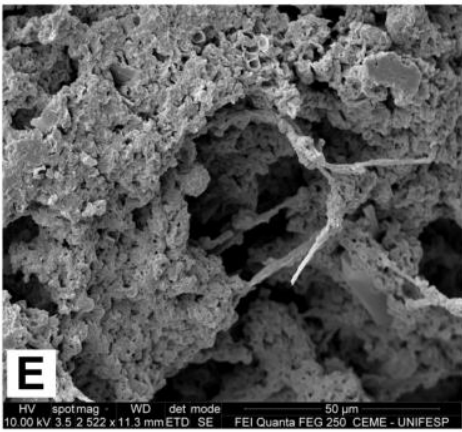

Outer face

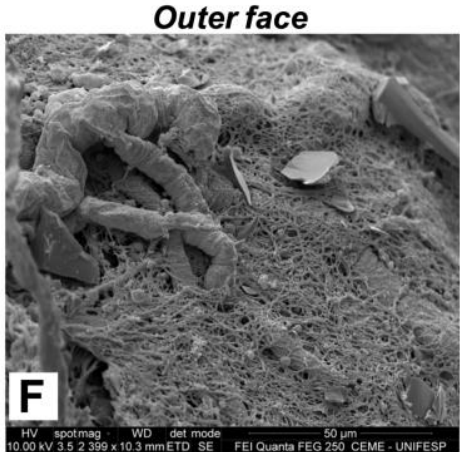

Figure 4: SEM images of DBM. (A) Internal region of DBM showing fibers of different lengths and thicknesses. (B) Internal region of DBM showing holes. Scale $=10 \mu \mathrm{m}$. Scale $=50 \mu \mathrm{m}$. (C) Internal region of DBM 24h after recellularization with Neuro2a cell. Scale $=50 \mu \mathrm{m}$. (D) Internal region of DBM $24 \mathrm{~h}$ focusing on one cell. Scale $=10 \mu \mathrm{m}$. (E) Internal region of DBM showing the preservation of grooves. Scale $=50 \mu \mathrm{m}$. (F) External region of DBM showing the presence of a vessel. Scale $=50 \mu \mathrm{m}$.

\section{DBM maintained cells in an undifferentiated state}

After injecting suspensions of Neuro2a cells into the DBM, they were incubated at $37^{\circ} \mathrm{C}$ and $5 \% \mathrm{CO}_{2}$ for 24 and $72 \mathrm{~h}$. After fixing and performing the histological sections of the DBM, it was possible to find Neuro2a cells by immunostaining (Figure 5A and F), showing that the scaffold was able to maintain the cells alive and undifferentiated. There was no evidence of neuronal differentiation of Neuro2a cells (more evident TUBB3 marking and neurite 
extension), confirming that during these periods of incubations the scaffold did not induce neuronal differentiation, as observed by SEM (Figure 4).

24h
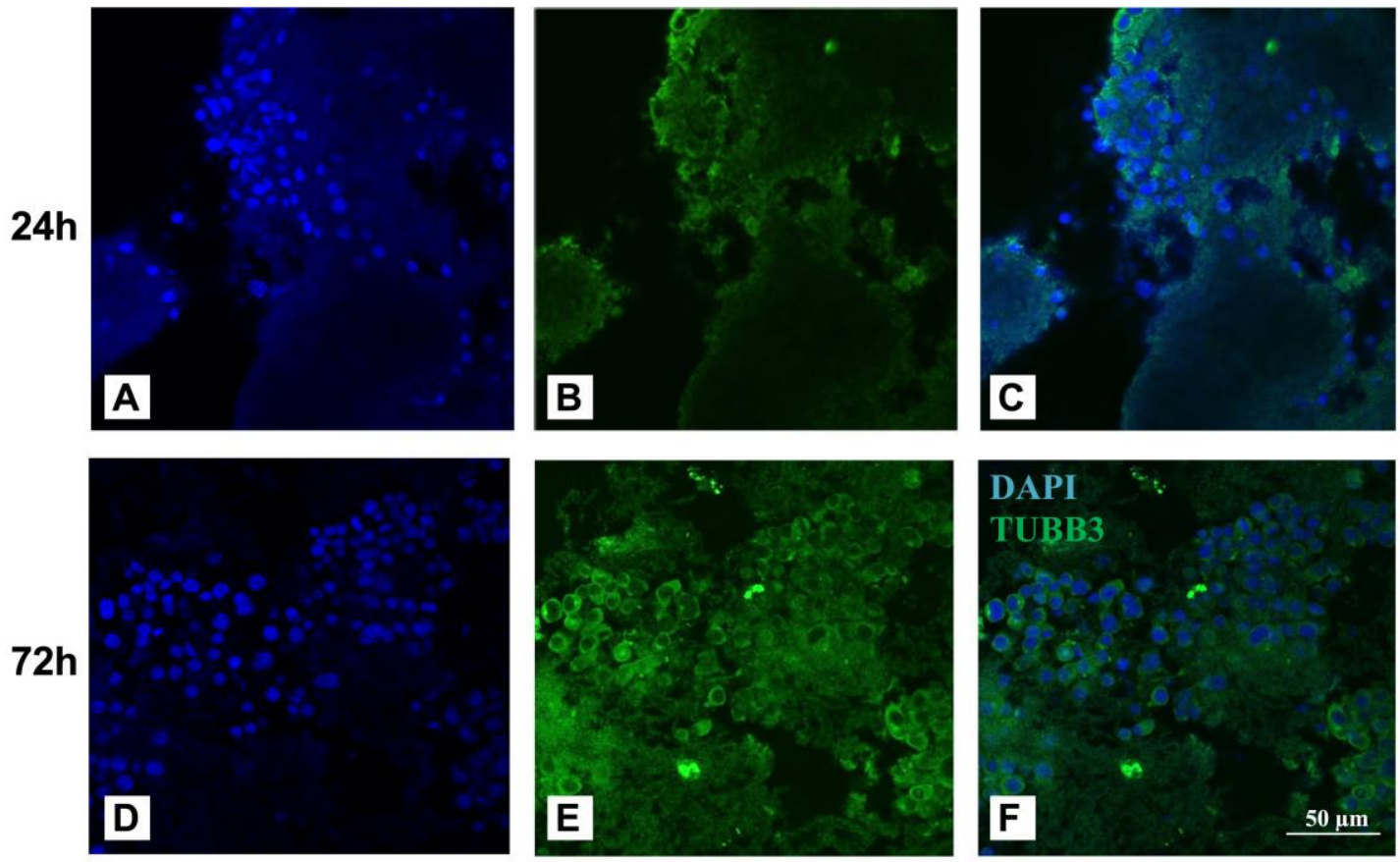

Figure 5: Neuro2a cells cultures inside DBM for 24h (A-C), 72h (D-F). Marker for mature neuron (Tubulin beta 3; TUBB3; green) (B,E), nuclear staining (DAPI; blue) $(A, D)$ and merges of images $(\mathrm{C}, \mathrm{F})$. Scale $=50 \mu \mathrm{m}$.

\section{DISCUSSION}

In the present work, we investigated the use of ECM scaffolds from murine brains (DBM) as a platform for repopulation of decellularized tissue. Our results showed a fast and straightforward method based on freezing and thawing murine brains followed by soaking them in a $1 \%$ SDS solution for complete decellularization. This concentration was able to decellularize the brains with minimal residues of DNA reminiscent and preserving structures as the basement membrane, collagen IV, as well as adhesive proteins such as laminin. For the first time it was shown that a simple and rapid process involving just one chemical reagent could successfully decellularize murine brains. 
The ECM has a crucial role in the development and repair of tissues. It offers support to cellular organization, and participates of cell growth and proliferation by the interaction with growth factors and cytokines (34-37). Therefore, the preservation of the ECM after the decellularization process showed here is very important to help cell reorganization after repopulation. In this work, after establishing a novel simple method for complete murine brain decellularization, and preservation of ECM structures, we also showed that the remaining scaffold (DBM) is biocompatible, being capable of maintaining Neuro2a cells alive for $72 \mathrm{~h}$.

Few data regarding brain decellularization demonstrated complete decellularization with different methods ${ }^{38}$. Decellularization regarding the regeneration of the CNS has been attempted by different techniques such as: detergent ${ }^{39}$, detergent-enzimatic combination ${ }^{40}$, enzymatic ${ }^{41}$, and physical methods such as freezing and thawing and applying high hydrostatic pressure 42 . Each of these protocols have their advantages and limitations. Notwithstanding, there are only a few studies concerning brain decellularization and its use for repopulation or as a scaffold.

Zhu et al. decellularized mice cerebellum and the resulting cerebellar scaffold retained neurosupportive proteins and growth factors and when tested with neural stem cells (NSCs) in vitro, was found to be cytocompatible ${ }^{43}$. De Waele et al. also decellularized mice cerebellum and the survival and growth of seeded NSC cells was monitored for 1-7 weeks in culture, indicating the ability of the acellular brain sections to support sustained ex vivo growth of NSC retaining their NSC phenotype (undifferentiated) ${ }^{44}$. These data corroborate with the results that we have described in this study, although we used a faster more 
straightfoward method for decellularization and fast-growing mouse neuroblastoma cell line (Neuro2a) with a shorter period of incubation (24 and $72 \mathrm{~h}$ ) inside the decellularized brain scaffolds (DBM). It is worth mentioning that this study has some limitations and shall be continued analyzing the composition of the DBM in more depth.

\section{CONCLUSION}

Decellularized scaffolds derived from an organ can be used as a platform to understand decellularization and cell repopulation. Although further studies are still required to validate the present data in translational models, our results are promising due to the fact that we could fully decellularize murine brains with a fast (24 $\mathrm{h}$ for complete decellularization) and simple (only one detergent and 3 freezing and thawing processes) method. The decellularization process also retained essential ECM proteins that are crucial for cell adhesion, migration, proliferation and consequently repopulation of the tissue. Lastly, the decellularized brain scaffold showed to be biocompatible, as neural cells were injected inside their structure and could be identified by immunohistochemistry.

\section{AKNOWLEDGEMENTS}

This work was supported by Fundação de Amparo à Pesquisa do Estado de São Paulo - FAPESP (2012/00652-5), Conselho Nacional de Desenvolvimento Científico e Tecnológico - CNPq (402319/2013-3; 465656/2014-5), and Coordenação de Aperfeiçoamento de Pessoal de Nível Superior - CAPES (Finance Code 001). 


\section{References}

1. Huang, Z., Godkin, O. \& Schulze-Tanzil, G. The challenge in using mesenchymal stromal cells for recellularization of decellularized cartilage. Stem Cell Rev. Reports 13, 50-67 (2017).

2. Kuevda, E. et al. Modification of Rat Lung Decellularization Protocol Based on Dynamic Conductometry of Working Solution. Bull. Exp. Biol. Med. 162, (2017).

3. Taylor, D. A., Parikh, R. B. \& Sampaio, L. C. Bioengineering hearts: simple yet complex. Curr. stem cell reports 3, 35-44 (2017).

4. Hassanein, W. et al. Recellularization via the bile duct supports functional allogenic and xenogenic cell growth on a decellularized rat liver scaffold. Organogenesis 13, 16-27 (2017).

5. Ott, H. C. Perfusion decellularization of discarded human kidneys: a valuable platform for organ regeneration. Transplantation 99, 1753 (2015).

6. Takebe, T., Zhang, B. \& Radisic, M. Synergistic engineering: organoids meet organs-ona-chip. Cell Stem Cell 21, 297-300 (2017).

7. Morizane, R. et al. Nephron organoids derived from human pluripotent stem cells model kidney development and injury. Nat. Biotechnol. 33, 1193 (2015).

8. Takasato, M. \& Little, M. H. Making a kidney organoid using the directed differentiation of human pluripotent stem cells. in Organ Regeneration 195-206 (Springer, 2017).

9. Lancaster, M. A. \& Knoblich, J. A. Organogenesis in a dish: modeling development and disease using organoid technologies. Science (80-. ). 345, 1247125 (2014).

10. Lancaster, M. A. et al. Cerebral organoids model human brain development and microcephaly. Nature 501, 373 (2013).

11. Qian, X. et al. Brain-region-specific organoids using mini-bioreactors for modeling ZIKV exposure. Cell 165, 1238-1254 (2016).

12. Takasato, M. et al. Kidney organoids from human iPS cells contain multiple lineages and model human nephrogenesis. Nature 526, 564 (2015).

13. Chow, T., Whiteley, J. \& Rogers, I. M. Decellularizing and Recellularizing Adult Mouse Kidneys. in Kidney Organogenesis 169-184 (Springer, 2019).

14. Regalado-Santiago, C., Juárez-Aguilar, E., Olivares-Hernández, J. D. \& Tamariz, E. Mimicking neural stem cell niche by biocompatible substrates. Stem Cells Int. 2016, (2016).

15. Wong, M. L., Wong, J. L., Vapniarsky, N. \& Griffiths, L. G. In vivo xenogeneic scaffold fate is determined by residual antigenicity and extracellular matrix preservation. Biomaterials 92, 1-12 (2016).

16. Qiao, W. et al. Sequential hydrophile and lipophile solubilization as an efficient method for decellularization of porcine aortic valve leaflets: Structure, mechanical property and biocompatibility study. J. Tissue Eng. Regen. Med. 12, e828-e840 (2018).

17. Seyler, T. M. et al. The development of a xenograft-derived scaffold for tendon and ligament reconstruction using a decellularization and oxidation protocol. Arthrosc. J. Arthrosc. Relat. Surg. 33, 374-386 (2017).

18. Poornejad, N. et al. The impact of decellularization agents on renal tissue extracellular matrix. J. Biomater. Appl. 31, 521-533 (2016).

19. Aguiari, P. et al. In vitro comparative assessment of decellularized bovine pericardial patches and commercial bioprosthetic heart valves. Biomed. Mater. 12, 15021 (2017).

20. Momtahan, N. et al. Using hemolysis as a novel method for assessment of cytotoxicity 
and blood compatibility of decellularized heart tissues. Asaio J. 62, 340-348 (2016).

21. Santoro, R. et al. Feasibility of pig and human-derived aortic valve interstitial cells seeding on fixative-free decellularized animal pericardium. J. Biomed. Mater. Res. Part B Appl. Biomater. 104, 345-356 (2016).

22. Shojaie, S. et al. Acellular lung scaffolds direct differentiation of endoderm to functional airway epithelial cells: requirement of matrix-bound HS proteoglycans. Stem Cell Reports 4, 419-430 (2015).

23. Sambi, M. et al. Acellular mouse kidney ECM can be used as a three-dimensional substrate to test the differentiation potential of embryonic stem cell derived renal progenitors. Stem Cell Rev. Reports 13, 513-531 (2017).

24. Chani, B., Puri, V., Sobti, R. C., Jha, V. \& Puri, S. Decellularized scaffold of cryopreserved rat kidney retains its recellularization potential. PLoS One 12, e0173040 (2017).

25. $\mathrm{Xu}, \mathrm{K}$. et al. Efficient decellularization for tissue engineering of the tendon-bone interface with preservation of biomechanics. PLoS One 12, e0171577 (2017).

26. Napierala, $\mathrm{H}$. et al. Engineering an endocrine neo-pancreas by repopulation of a decellularized rat pancreas with islets of Langerhans. Sci. Rep. 7, 41777 (2017).

27. Liu, W.-Y. et al. Xenogeneic Decellularized Scaffold: A Novel Platform for Ovary Regeneration. Tissue Eng. Part C Methods 23, 61-71 (2017).

28. Roth, S. P. et al. Automated freeze-thaw cycles for decellularization of tendon tissue-a pilot study. BMC Biotechnol. 17, 13 (2017).

29. Pu, L. et al. Determining the optimal protocol for preparing an acellular scaffold of tissue engineered small-diameter blood vessels. J. Biomed. Mater. Res. Part B Appl. Biomater. 106, 619-631 (2018).

30. Butter, A. et al. Evolution of graft morphology and function after recellularization of decellularized rat livers. J. Tissue Eng. Regen. Med. 12, e807-e816 (2018).

31. Bao, J. et al. Construction of a portal implantable functional tissue-engineered liver using perfusion-decellularized matrix and hepatocytes in rats. Cell Transplant. 20, 753-766 (2011).

32. Shirakigawa, N., Takei, T. \& ljima, H. Base structure consisting of an endothelialized vascular-tree network and hepatocytes for whole liver engineering. J. Biosci. Bioeng. 116, 740-745 (2013).

33. Lattouf, R. et al. Picrosirius red staining: a useful tool to appraise collagen networks in normal and pathological tissues. J. Histochem. Cytochem. 62, 751-758 (2014).

34. Crapo, P. et al. Biologic scaffolds composed of central nervous system extracellular matrix. Biomaterials 33, (2012).

35. Wu, Y. et al. Implantation of Brain-derived Extracellular Matrix Enhances Neurological Recovery after Traumatic Brain Injury. Cell transplantation (2016). doi:10.3727/096368916X692744

36. Vorotnikova, E. et al. Extracellular matrix-derived products modulate endothelial and progenitor cell migration and proliferation in vitro and stimulate regenerative healing in vivo. Matrix Biol. 29, 690-700 (2010).

37. Bonnans, C., Chou, J. \& Werb, Z. Remodelling the extracellular matrix in development and disease. Nat. Rev. Mol. Cell Biol. 15, 786-801 (2014).

38. Wang, H. et al. Decellularization technology in CNS tissue repair. Expert Rev. Neurother. 15, 493-500 (2015).

39. Sondell, M., Lundborg, G. \& Kanje, M. Regeneration of the rat sciatic nerve into allografts made acellular through chemical extraction. Brain Res. 795, 44-54 (1998). 
40. Ribatti, D. et al. Angiogenic response induced by acellular brain scaffolds grafted onto the chick embryo chorioallantoic membrane. Brain Res. 989, 9-15 (2003).

41. Grauss, R. W. et al. Histological evaluation of decellularised porcine aortic valves: matrix changes due to different decellularisation methods. Eur. J. cardio-thoracic Surg. 27, 566-571 (2005).

42. Gilpin, A. \& Yang, Y. Decellularization Strategies for Regenerative Medicine: From Processing Techniques to Applications. Biomed Res. Int. 2017, 9831534 (2017).

43. Zhu, T. et al. An acellular cerebellar biological scaffold: preparation, characterization, biocompatibility and effects on neural stem cells. Brain Res. Bull. 113, 48-57 (2015).

44. De Waele, J. et al. 3D culture of murine neural stem cells on decellularized mouse brain sections. Biomaterials 41, 122-131 (2015). 
bioRxiv preprint doi: https://doi.org/10.1101/680702; this version posted June 24,2019 . The copyright holder for this preprint (which was not certified by peer review) is the author/funder, who has granted bioRxiv a license to display the preprint in perpetuity. It is made available under aCC-BY-ND 4.0 International license.

\section{GRAFICAL ABSTRACT}

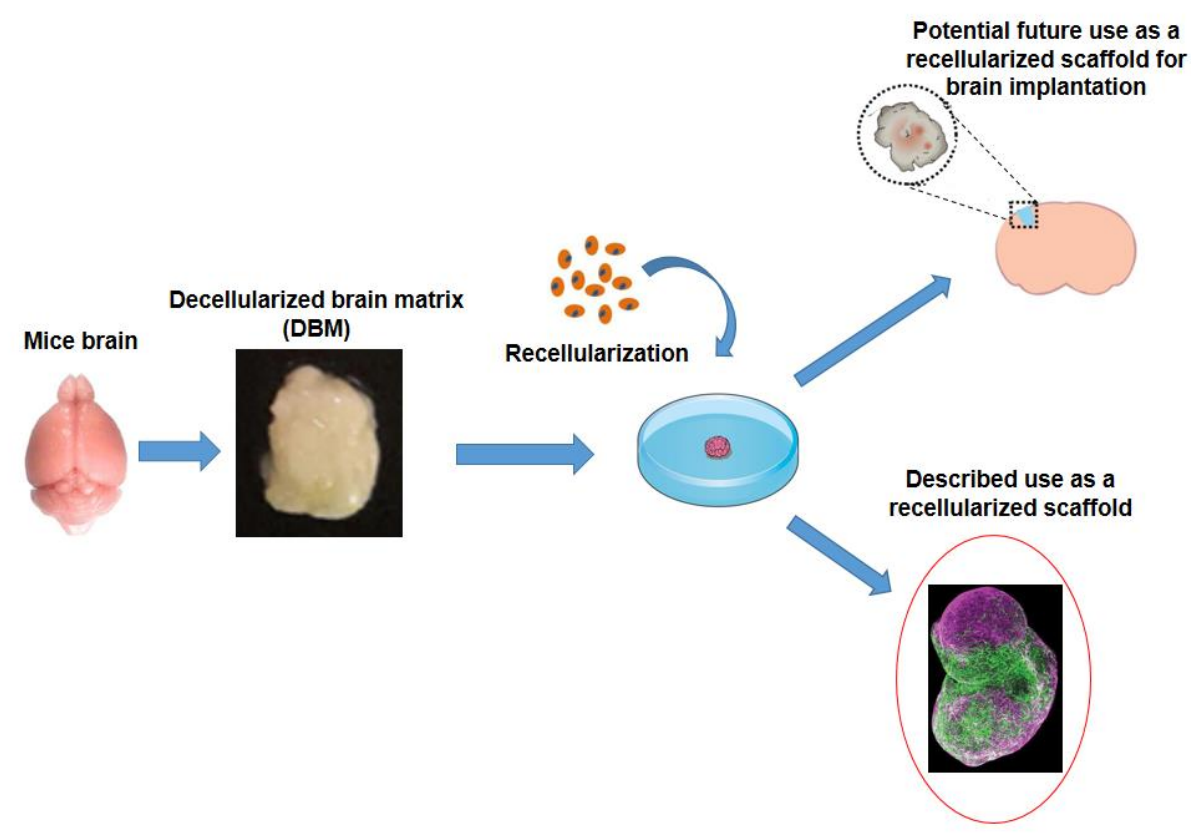

